\title{
SELETIVIDADE DE ARMADILHAS E ATRATIVOS NA CAPTURA DE PEQUENOS PEIXES E CRUSTÁCEOS EM AMBIENTES ESTUARINOS
}

\author{
POSSAMAI, B. ${ }^{1 *}$; ROSA, L. C. ${ }^{2} \&$ CORRÊA, M. F. M. ${ }^{1}$ \\ 1 - Laboratório de Ictiologia, Centro de Estudos do Mar, Universidade Federal do Paraná \\ 2 - Núcleo de Engenharia de Pesca, Centro de Ciências Biológicas e da Saúde, Universidade Federal \\ de Sergipe \\ *Corresponding author: biancapossamai@hotmail.com
}

\begin{abstract}
Possamai, B.; Rosa, L. C. \& Corrêa, M. F. M. (2014) Traps and baits selectivity on the capture of small fishes and crustaceans at estuarine environments. Braz. J. Aquat. Sci. Technol. 18(2): 11-17. elSSN 1983-9057. DOI: 10.14210/ bjast.v18n2.p11-17 In this study we tested capture efficiency of two of plastic bottle traps and four kinds of baits (fresh cow liver, broken corn, meat flavored dog food and commercial bait for amateur fishing) for small crustaceans and fishes at estuarine environments (saltmarshes and tidal creeks). Smaller trap opening sizes mainly limited the capture of organisms with hard exoskeleton such as crabs. There were no significant differences in number of species, abundance and/or biomass of captured organisms among baits. However, a bait/feeding behavior relationship was observed. The traps and baits were efficient in capturing small fishes and crustaceans, and therefore can be applied as a complementary method in ichthyological and crustaceans surveys at estuarine shallow water environments. However, in order to increase capture efficiency we recommend to use mixed baits, which best represent the trophic group diversity present in the study area.
\end{abstract}

Key words: Methodological evaluation; sampling; ichthyofauna; crustaceans; saltmarshes; tidal creeks.

\section{INTRODUÇÃO}

Os métodos de amostragem de peixes e crustáceos são separados, segundo a participação do operador, em ativos e passivos (Nielsen \& Johnson, 1983). A amostragem passiva é realizada utilizando-se petrechos sem a intervenção direta do coletor, causando uma menor perturbação ao ambiente, contrariamente ao método ativo, que consiste na captura utilizando-se petrechos que perturbam o ambiente, seja pela movimentação dos coletores ou pela alteração de microhábitats (Ribeiro \& Zuanon, 2006).

Dentre os métodos passivos existentes, o encarceramento consiste em dispositivos de armadilha que capturam os organismos que entram em uma área delimitada através de um ou mais funis - ou aberturas em forma de $\mathrm{V}$ - e que, uma vez lá dentro, não conseguem encontrar um caminho para escape (Hubert, 1996).

No litoral norte de São Paulo e sul do Rio de Janeiro as armadilhas são utilizadas pelos pescadores artesanais na captura de peixes em áreas de costões rochosos e parcéis. De maneira semelhante, no nordeste brasileiro estas também são utilizadas na captura de peixes e crustáceos em ambientes recifais (Sanches \& Sebastiani, 2009) e como amostradores de fauna em ambientes estuarinos (Carvalho \& Couto, 2011) e dulceaquicolas (Teixeira \& Couto, 2012).

Métodos passivos de amostragem foram previamente estudados nos aspectos da avaliação do melhor tempo de submersão das armadilhas e da eficiência dos atrativos na captura de peixes (Sanches \& Sebastiani, 2009); dos tipos de petrecho (armadilhas e redes) na captura do siri-azul (Atar et al., 2002); da seletividade das armadilhas quanto ao tamanho dos peixes (Bohnsack et al., 1989; Gobert, 1998); da comparação entre métodos passivos e ativos na amostragem de peixes (Uieda \& Uieda, 2001; Ribeiro \& Zuanon, 2006); entre formatos de armadilhas (Collins, 1990); e descrição sobre vantagens e desvantagens de métodos passivos e ativos de amostragem de peixes (Uieda \& Castro, 1999). São métodos frequentemente utilizados como amostradores complementares, principalmente em ambientes estuarinos rasos (Rozas \& Minello, 1997).

As vantagens da utilização de armadilhas são a facilidade de confecção, de operação e de uso, permitindo a utilização manual sem requerimento de máquinas e muito treino (Hubert, 1996), baixo custo operacional, além de permitir o seu uso mesmo em condições climáticas desfavoráveis, uma vez que podem permanecer instaladas no meio por prolongados períodos (Atar et al., 2002). Outra vantagem da utilização desse método é que os animais ficam vivos, portanto, indivíduos juvenis e fêmeas ovígeras podem ser devolvidos logo após a captura (Atar et al., 2002). Porém, esses métodos são mais seletivos e menos eficientes do que os ativos quanto à riqueza $\mathrm{e}$ abundância das espécies capturadas (Uieda \& Castro, 1999; Ribeiro \& Zuanon, 2006), sendo ainda que, a eficiência de captura dependerá principalmente do tipo de atrativo (i.e. tipo de isca) utilizado, os quais deverão corresponder às características tróficas das espécies alvo (Hubert, 1996; Uieda \& Castro, 1999; Sanches \& Sebastiani, 2009; Rocha, 2010). Contudo, os métodos passivos são considerados importantes 
nos estudos de ambientes estuarinos rasos, que incluem gramíneas emergentes, vegetação aquática submersa, mangues e bancos arenosos, cujas baixas profundidades e vegetação abundante dificultam as amostragens de pequenos peixes e crustáceos (Rozas \& Minello, 1997).

Deste modo, o presente estudo teve como objetivo verificar a eficiência de dois tipos de armadilhas confeccionadas com garrafas "PET" e de quatro tipos de atrativos utilizados como iscas na captura de pequenos peixes e crustáceos em ambientes estuarinos, a fim de contribuir para a escolha do método a ser empregada em futuros trabalhos de espécies alvo e amostragens em ambientes estuarinos rasos.

\section{MATERIAIS E MÉTODOS}

\section{Área de estudo}

A Gamboa do Perequê está localizada próxima à desembocadura da Baía de Paranaguá (Figura 1). Apresenta baixa profundidade, atingindo na maré mais alta $2,8 \mathrm{~m}$ e, ao longo de seus $2,6 \mathrm{~km}$ de extensão, drena manguezais formados principalmente por Laguncularia racemosa (L.) C.F.Gaertn e áreas de restingas (Lana et al., 1989). O fluxo de água é regido pelas marés e a salinidade sofre variações, tanto pela pluviosidade quanto pelas marés. Suas águas são mais salinas no inverno (até 33) e na época de chuvas apresenta salinidade em torno de um (Soares et al., 1996). Nas margens da gamboa, geralmente nos meandros à direita, encontram-se bancos colonizados por Spartina alterniflora Loisel., que caracterizam as marismas locais de faixas monoespecíficas, sempre estreitas, pouco densas e baixas, com no máximo $50 \mathrm{~cm}$ de altura (Soares et al., 1996).

\section{Experimento}

Nesse experimento foram utilizados dois tipos de covos (denominados de grande e pequeno) e quatro atrativos (fígado bovino crú, quirera grossa, ração para cães sabor carne e massinha preparada para pesca de lambari). Os covos foram confeccionados a partir de garrafas "PET" transparentes, de $2 \ell$ de capacidade, que tiveram sua parte superior cortada e invertida para formar um funil. A principal diferença entre os tamanhos dos covos residia na abertura do funil, que no pequeno apresentava a abertura fixa de $25 \mathrm{~mm}$ de diâmetro, enquanto que o grande apresentava cortes perpendiculares, que permitiam a expansão da abertura até $55 \mathrm{~mm}$. Os atrativos foram colocados soltos dentro das armadilhas de forma a ficarem disponíveis para os animais, sendo que a cada despesca ainda havia uma grande quantidade destes em cada covo. Para cada combinação de covo e atrativo foram realizadas três réplicas (cada tamanho com quatro iscas), totalizando 24 unidades (Figura 2).

O experimento foi conduzido entre os meses de maio e julho de 2009, em dois ambientes distintos, em

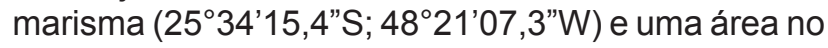
canal principal $\left(25^{\circ} 34^{\prime} 23,7^{\prime \prime} \mathrm{S}\right.$; 48 $\left.21^{\prime} 04,1^{\prime \prime} \mathrm{W}\right)$ (Figura 1), com duas repetições em cada ambiente. Em cada uma das situações os covos foram aleatoriamente distribuídos, permanecendo no local por 24 horas. As despescas foram realizadas a cada 12 horas (correspondentes aos picos da maré baixa) e, após esse período, os organismos capturados eram transferidos para sacos plásticos devidamente identificados, fixados e transportados ao laboratório para posterior identificação específica de acordo com Figueiredo \& Menezes (1978, 2000), Menezes \& Figueiredo (1980) para peixes e Melo $(1996,2003)$ para crustáceos. A autorização para coleta do material foi fornecida pelo

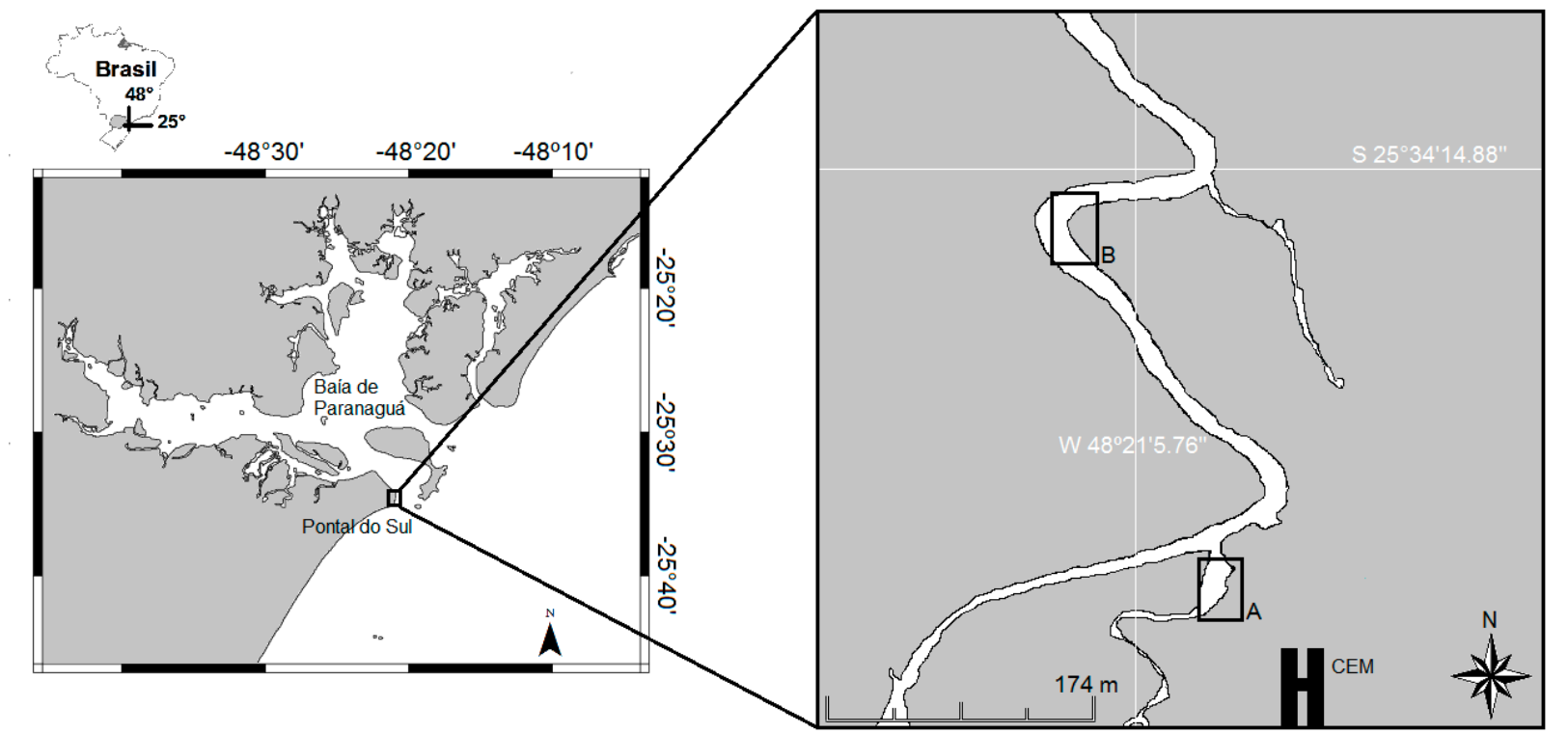

Figura 1 - Area de estudo. Baía de Paranaguá com recorte mostrando a Gamboa do Perequê. (A) Area do canal amostrada; (B) banco de Spartina alterniflora. "CEM" representa o Centro de Estudos do Mar, da Universidade Federal do Paraná. 


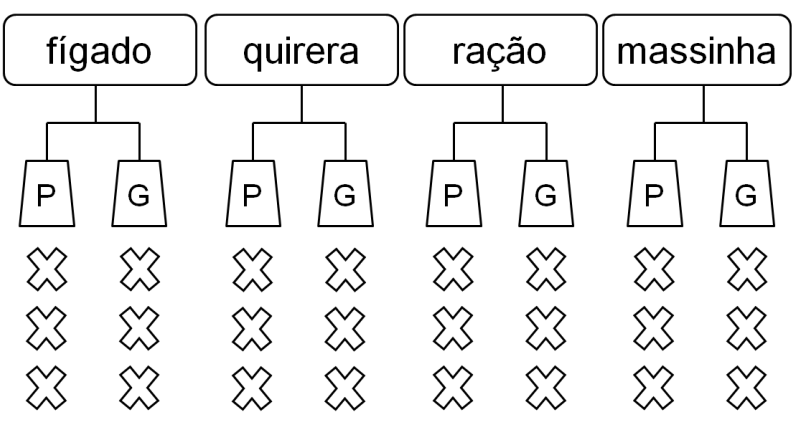

Figura 2 - Esquema da amostragem. Losangos indicam as armadilhas $(P=$ pequenas e $G=$ grandes $)$ e " $x$ " indicam as repetições.

IBAMA (Autorização para coleta de material zoológico - SISBIO/11453).

\section{Análises}

Todos os indivíduos capturados foram medidos com o auxílio de um ictiômetro (comprimento total para os peixes) ou de um paquímetro digital (largura da carapaça para os siris e caranguejos e comprimento da carapaça para os camarões) e pesados numa balança digital (precisão de 0,001g).

Como a principal causa de variação entre os tamanhos de covos estava relacionada à sua abertura e pelo fato de que essa característica poderia afetar o tamanho e/ou biomassa dos indivíduos capturados, essas duas variáveis foram comparadas, independente do período e do atrativo, através Teste-t separadamente para cada espécie. A priori foi realizado um teste qui-quadrado para verificar se as diferenças observadas nas frequências de captura (independente do tamanho do covo e do atrativo) entre os dois ambientes estudados foram significativas. Como as diferenças nas frequências de captura entre os ambientes não foram significativas estes foram analisados conjunta- mente. As diferenças entre o número de espécies, as frequências de indivíduos capturados e os tipos de atrativos foram testados pelo teste não paramétrico $F$ e a biomassa total capturada, também em relação aos atrativos, foi testada através de uma análise de variância, sendo que os dados foram testados a priori para homocedasticidade (Teste de Cochran) e normalidade da variância (Teste de Kolmogorov-Smirnov) (Zar, 1996). Possíveis relações entre as espécies capturadas e o tipo de atrativo foram verificadas através de uma análise de correspondência (Zuur et al., 2007).

\section{RESULTADOS}

No total foram capturados 166 exemplares, distribuídos em cinco espécies de crustáceos e sete espécies de peixes (Tabela 1). A espécie mais abundante foi o camarão-fantasma Palaemon pandaliformis (Stimpson, 1871) $(26,5 \%)$, seguida do peixe-rei Atherinella brasiliensis (Quoy \& Gaimard, 1825) (24,7\%), do amborê Bathygobius soporator (Valenciennes, 1837) (12,65\%) e do siri-azul Callinectes danae Smith, 1869 (10,84\%). As demais espécies apresentaram frequência inferior a $10 \%$.

A influência do tamanho da abertura do covo sobre o tamanho e/ou biomassa dos organismos capturados mostrou-se significativa apenas para o siri Callinectes danae e para o barrigudinho Poecilia vivipara Bloch \& Schneider, 1801, uma vez que indivíduos de maior tamanho e/ou peso foram capturados nos covos com aberturas maiores (covos grandes) (Tabela 1).

Em relação aos atrativos, a maior frequência de indivíduos foi capturada com a ração (Figura 3). No entanto, não foi observado nenhuma diferença

Tabela 1 - Número de indivíduos $(\mathrm{N})$, tamanho e peso (média \pm desvio padrão) das espécies capturadas nos dois tipos de covos, independente do tipo de atrativo. Valores em destaque correspondem às diferenças significativas pelo Teste-t (nt não testado; $\left.{ }^{*} p<0,05\right)$.

\begin{tabular}{|c|c|c|c|c|c|c|}
\hline \multirow{2}{*}{ Espécies } & \multicolumn{3}{|c|}{ Covo Pequeno } & \multicolumn{3}{|c|}{ Covo Grande } \\
\hline & $\mathrm{N}$ & Tamanho $(\mathrm{mm})$ & Peso $(g)$ & $\mathrm{N}$ & Tamanho $(\mathrm{mm})$ & Peso (g) \\
\hline \multicolumn{7}{|l|}{ Crustáceos } \\
\hline Armases rubripes ${ }^{\text {nt }}$ (Rathbun, 1897) & - & - & - & 3 & $4,18( \pm 2,09)$ & $0,08( \pm 0,12)$ \\
\hline Callinectes danae Smith, 1869 & 6 & $20,36( \pm 6,11)$ & $1,66( \pm 0,95)$ & 12 & $\underline{34,26( \pm 12,73)^{*}}$ & $8,02( \pm 6,83)^{*}$ \\
\hline Macrobrachium acanthurus ${ }^{\text {nt }}$ (Wiegmann, 1836) & 1 & 12,80 & 1,93 & - & - & - \\
\hline Penaeidae (misis) $^{\text {nt }}$ (Rafinesque, 1815) & - & - & - & 1 & 1,86 & 0,01 \\
\hline Palaemon pandaliformis (Stimpson, 1871) & 17 & $4,98( \pm 0,79)$ & $0,22( \pm 0,09)$ & 27 & $4,32( \pm 1,28)$ & $0,17( \pm 0,11)$ \\
\hline Panopeus sp. ${ }^{\mathrm{nt}} \mathrm{H}$. Milne Edwards, 1834 & 1 & 17,85 & 2,51 & - & - & - \\
\hline \multicolumn{7}{|l|}{ Peixes } \\
\hline Atherinella brasiliensis (Quoy \& Gaimard, 1825) & 30 & $95,07( \pm 9,66)$ & $5,28( \pm 1,49)$ & 11 & $92,27( \pm 5,62)$ & $4,70( \pm 0,83)$ \\
\hline Bathygobius soporator (Valenciennes, 1837) & 11 & $67,45( \pm 34,76)$ & $6,61( \pm 7,59$ & 10 & $54,90( \pm 37,76)$ & $5,06( \pm 8,55)$ \\
\hline Dormitator maculatus (Bloch, 1792) & 2 & $56,00( \pm 2,83)$ & $0,89( \pm 0,07)$ & 3 & $72,00( \pm 53,73)$ & $9,09( \pm 15,20)$ \\
\hline Gobionellus oceanicus ${ }^{\text {nt }}$ (Pallas, 1770) & 1 & 34,00 & 0,10 & - & - & - \\
\hline Poecilia vivipara Bloch \& Schneider, 1801 & 4 & $40,75( \pm 5,19)$ & $0,86( \pm 0,34)$ & 4 & $\underline{54,25( \pm 9,50)^{*}}$ & $1,91( \pm 0,91)$ \\
\hline Sphoeroides greeleyi ${ }^{\text {nt }}$ Gilbert, 1900 & 1 & 110,00 & 28,73 & 8 & $84,75( \pm 17,16)$ & $13,71( \pm 7,60)$ \\
\hline Sphoeroides testudineus (Linnaeus, 1758) & 5 & $85,00( \pm 21,77)$ & $13,42( \pm 8,35)$ & 8 & $65,50( \pm 16,51)$ & $6,03( \pm 5,00)$ \\
\hline
\end{tabular}




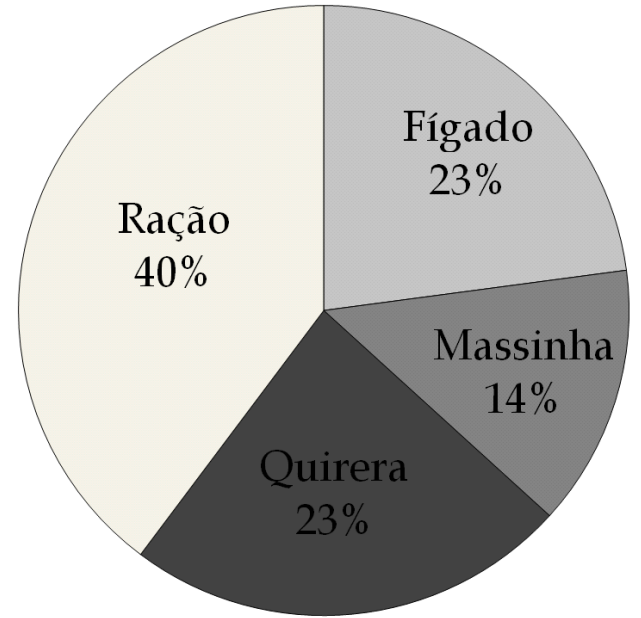

Figura 3 - Proporção do total de indivíduos capturados em cada um dos atrativos utilizados na Gamboa do Perequê - PR.

significativa no número médio de espécies $\left(F_{(3,92)}=1,61\right.$; $p=0,19)$, no número médio de indivíduos $\left(F_{(3,92)}^{(3,92)}=1,91\right.$; $p=0,13)$ nem na biomassa total $\left(F_{(3,92)}=3,01 ; p=0,05\right)$ capturada entre cada um dos atrativos (Figura 4).

As preferências das espécies capturadas por algum dos atrativos utilizados podem ser visualizadas na Figura 5 , que mostra a associação do peixe-rei $A$. brasiliensis à ração e do crustáceo $P$. pandaliformis à quirera. Já as capturas dos baiacus $S$. testudineus (Linnaeus, 1758) e S. greeleyi Gilbert, 1900 e do siri C. danae foram associadas à utilização do fígado como atrativo.

\section{DISCUSSÃO}

As armadilhas confeccionadas com garrafas "PET" mostraram-se eficientes na captura de peixes e crustáceos de pequeno porte em áreas estuarinas rasas. Apesar da amostragem ter sido realizada em curto espaço de tempo, foram capturadas 12 espécies de hábitos distintos. Dados sobre a carcinofauna local são inexistentes, no entanto, Pesserl (2007), testando a seletividade de redes de emalhe nessa mesma gamboa, registrou 24 espécies de peixes, sendo que das sete coletadas no presente estudo, o referido autor encontrou apenas três (S. greeleyi, S. testudineus e $A$. brasiliensis). Já captura de espécies crípticas como os gobídeos ( $G$. oceanicus e $B$. soporator) e também daqueles de menor tamanho e mais comumente encontrados em ambientes dulcícolas (D. maculatus e $P$. vivipara) nos covos indicam sua seletividade, bem como a importância de sua utilização como métodos complementares de amostragem.

O aumento do tamanho do funil de entrada da armadilha, ao contrário do esperado, não ocasionou um aumento no tamanho corpóreo dos indivíduos capturados, exceto para P. vivipara e C. danae. Essa
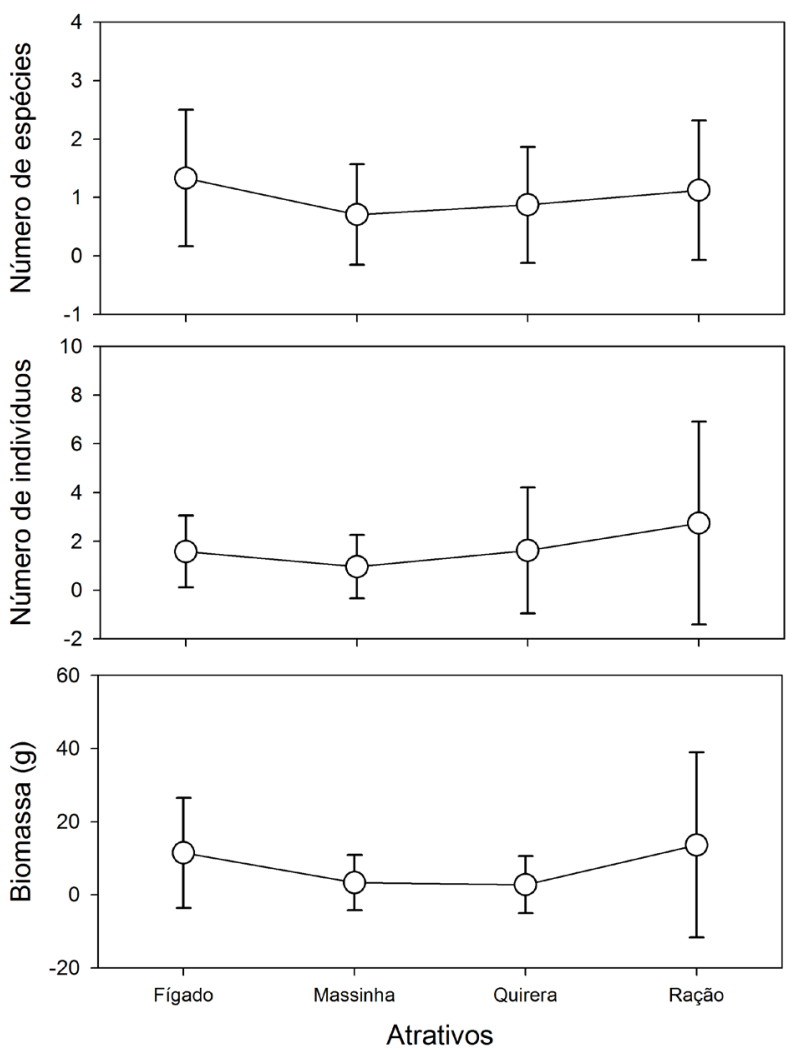

Figura 4 - Valores médios ( \pm 1 desvio padrão) do número de espécies, de indivíduos e de biomassa total capturado em cada tipo de atrativo na Gamboa do Perequê, Paraná.

seleção apresentada para o siri-azul pode ter ocorrido devido à carapaça rígida deste dificultar a passagem do animal pelo funil menor, de forma que foram encontrados indivíduos maiores de $50 \mathrm{~mm}$ apenas nos covos grandes. Comparando três tipos de armadilhas de diferentes dimensões para a captura do caranguejo-azul Callinectes sapidus Rathbun, 1896 na Turquia, Atar et al. (2002) perceberam que estas capturaram indivíduos de todos os tamanhos, mas em proporções diferentes. Importante ressaltar, porém, que todas as armadilhas utilizadas pelos autores tinham capacidade e abertura de funil maior do que as empregadas no presente estudo levando a acreditar que a seleção observada está relacionada ao tamanho da entrada do funil menor.

Apesar de ser comum na literatura trabalhos afirmando que o petrecho seleciona o tamanho dos animais capturados, é provável que o resultado obtido para os barrigudinhos deva-se ao acaso, uma vez que a captura de outras espécies de tamanhos corpóreos maiores que os observados para $P$. vivipara não foi afetada pelo tamanho da abertura do covo. Bohnsack et al. (1989) identificaram que o tamanho da malha das armadilhas utilizadas para a captura de peixes recifais 


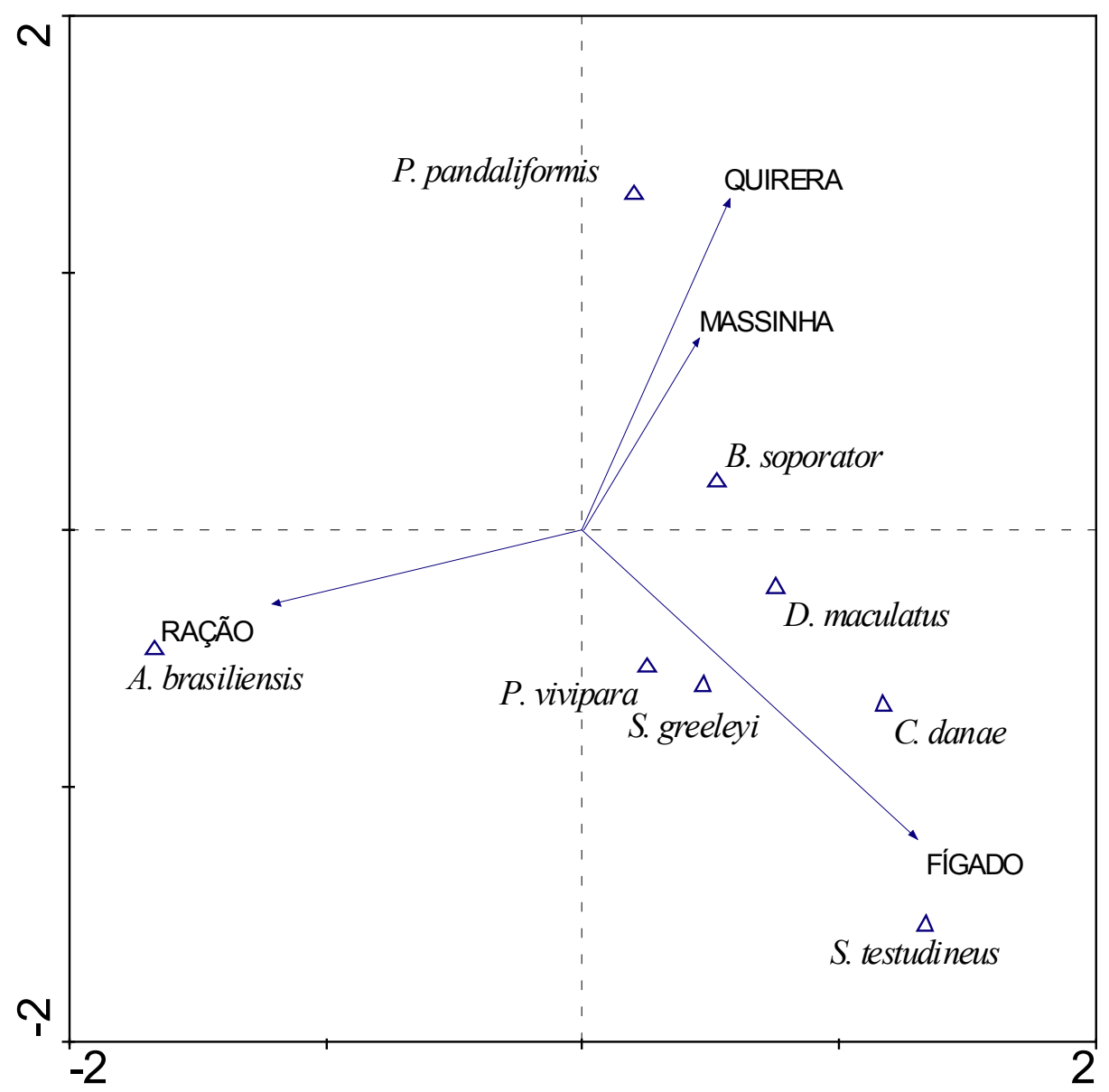

Figura 5 - Diagrama da Análise de Correspondência mostrando a relação entre as espécies capturadas e os atrativos utilizados na Gamboa do Perequê, Paraná.

na Flórida influencia na taxa de escape, uma vez que indivíduos menores são capazes de passar por malhas de grandes tamanhos. Verificaram, também, que não só o tamanho da malha, mas também a forma e o tamanho da abertura influenciam no comprimento dos peixes capturados. Em nosso experimento esse fato não foi observado, pois foram capturados indivíduos de tamanhos muito diferentes em ambas as aberturas de funil, possibilitando inferir que os animais menores não escaparam da armadilha, mesmo que seu tamanho corpóreo reduzido possibilitasse a fuga pelo funil.

Quanto a eficiência dos atrativos, embora tenha sido observada maior frequência de captura associada à ração, nenhum dos quatros atrativos se sobresaiu quanto ao número de espécies, abundância e/ou biomassa de indivíduos capturados. No entanto, cada um deles mostrou-se eficiente na atração de grupos específicos de organismos, baseado essencialmente em seus hábitos alimentares. Espécies como os baiacus $S$. testudineus e $S$. greeleyi, assim como o siri azul $C$. danae, foram essencialmente capturadas com fígado bovino crú como atrativo, evidenciando o hábito carnívoro oportunista dessas espécies (Branco \& Verani, 1997; Barros et al., 2010). Em um estudo realizado em Porto Rico verificou-se que $C$. danae alimentava-se principalmente de crustáceos, aumentando a ingestão de peixes ao longo do crescimento do indivíduo (Stoner \& Buchanan, 1990). Em Santa Catarina, Branco \& Verani (1997) encontraram moluscos, poliquetas e crustáceos como elementos básicos da dieta dessa espécie. Para C. sapidus, Oliveira et al. (2006) concluíram que a espécie é onívora oportunista, com uma tendência de predar invertebrados bentônicos, principalmente bivalves e gastrópodes, além de afirmar que os portunídeos são conhecidos como predadores vorazes. Da mesma forma, Barros et al. (2010) e Palacios-Sánchez \& Vega-Candejas (2010) encontraram grandes proporções de gastrópodes e bivalves nos estômagos de $S$. testudineus, enquanto que a dieta natural de $S$. greeleyi parece ser primariamente de ostracodas e isópodas, evidenciando-se um comportamento carnívoro bentófago (Corrêa \& Uieda, 2007).

O camarão carídeo Palaemon pandaliformis foi a espécie mais abundante, ocorrendo principalmente em covos iscados com quirera. Essa espécie caracteriza-se por ser de pequeno porte, detritívora, comumente encontrada junto à vegetação marginal 
de ambientes de águas doces e salobras, onde são capturadas com auxílio de peneiras e puçás (Müller et al., 1996; Lima \& Oshiro, 2002). Recentemente, Rocha (2010) testou a eficiência de peneiras e armadilhas na amostragem de decápodes, verificando uma maior captura de $P$. pandaliformis com o uso das últimas, as quais foram iscadas com coração de frango. Diferentemente, neste experimento, apenas um indivíduo de $P$. pandaliformis foi amostrado em covos com fígado bovino, sugerindo que a utilização de vísceras de animais não seja um eficiente atrativo para a captura dessa espécie. Portanto, mesmo sendo um método eficaz para amostragem desses carídeos, os covos devem ser iscados com atratores mais condizentes com as características detritívoras da espécie, como o caso da quirera utilizada neste trabalho.

O peixe-rei $A$. brasiliensis representou a segunda maior captura $(24,69 \%$ do total de indivíduos), com $92,68 \%$ dos indivíduos capturados em armadilhas contendo ração, demonstrando, assim, uma clara preferência por esse atrativo. Isto provavelmente está relacionado ao seu hábito alimentar suspensívoro, alimentando-se principalmente de fitoplâncton e detritos (Sazima, 1986). Atherinella brasiliensis é considerada uma espécie generalista e oportunista (Contente et al., 2010) e provavelmente foi beneficiada pela peculiaridade desse atrativo que, quando imerso, dissolvia-se dispersando na armadilha e na coluna d'água. Já as demais espécies não apresentaram um padrão nítido de preferência por algum dos atrativos testados, o que, em parte, foi resultante do baixo número de exemplares capturados.

\section{CONCLUSÃO}

Os resultados indicam que a utilização de covos como métodos complementares de coleta de pequenos peixes e crustáceos em áreas estuarinas rasas são eficientes, principalmente ao levarmos em conta o pequeno esforço amostral, além de ser de baixo custo e fácil confecção. Alterações na abertura do funil podem atuar de forma seletiva no tamanho dos organismos capturados, principalmente daqueles com exoesqueletos rígidos como os siris. Já que nenhum dos atrativos testados se sobressaiu quanto à eficiência de captura, aconselha-se a utilização de uma mistura contendo diversos atrativos, o que melhor reflete a diversidade trófica presente nesses ambientes.

\section{AGRADECIMENTOS}

Gostaríamos de agradecer aos oceanógrafos Bárbara Maichak de Carvalho e Augusto Luiz Ferreira
Júnior pelo auxílio em campo e durante as triagens do material.

\section{REFERÊNCIAS}

Atar, H.H.; Olmez, M.; Bekcan, S. \& Seçer, S. 2002. Comparison of three different traps for catching blue crab (Callinectes sapidus Rathbun 1896) in Beymelek Lagoon. Turk. J. Vet. Anim. Sci. 26(1): 1145-1150.

Barros, J.C.N.; Francisco, J.A.; Vasconcelos-Filho, A.L. \& Tenorio, D.O. 2010. Moluscos encontrados no trato digestivo de Sphoeroides testudineus (Linnaeus, 1758) (Teleostei: Tetraodontidae), no Canal de Santa Cruz, Itamaracá-Pernambuco, Brasil. REPesca. 5(1): 70-80.

Bohnsack, J.A.; Sutherland, D.L.; Harper, D.E.; McClellan, D.B.; Hulsbeck, M.W. \& Holt, C.M. 1989. The Effects of Fish Trap Mesh Size on Reef Fish Catch off Southeastern Florida. Mar. Fish. Rev. 51(2): 36-46.

Branco, J.O. \& Verani, J.R. 1997. Dinâmica da alimentação natural de Callinects danae Smith (Decapoda, Portunidae) na Lagoa da Conceição, Florianópolis, Santa Catarina, Brasil. Rev. Bras. Zool. 14(4): 1003-1018.

Carvalho, F. L. \& Couto, E. C. G. 2011. Environmental variables influencing the Callinectes (Crustacea, Brachyura, Portunidae) species distribution in a tropical estuary - Cachoeira River (Bahia, Brazil). J. Mar. Biol. Ass. U. K. 91(4): 793-800.

Collins, M.R. 1990. A comparison of three fish traps designers. Fish. Res. 9: 325-332.

Contente, R.F.; Stefanoni, M.F. \& Spach, H.L. 2010. Feeding ecology of the Brazilian silverside Atherinella brasiliensis (Atherinopsidae) in a sub-tropical estuarine ecosystem. J. Mar. Biol. Assoc. U.K. 91: 1197-1205. doi:10.1017/ S0025315410001116.

Corrêa, M. O. A. \& Uieda, V. S. 2007. Diet of the ichthyofauna associated with marginal vegetation of a mangrove forest in southeastern Brazil. Iheringia, Sér. Zool., Porto Alegre, 97(4):486-497.

Figueiredo, J.L. de \& Menezes, N.A. 1978. Manual de peixes marinhos do sudeste do Brasil - Volume II (Teleostei 1). São Paulo: Museu de Zoologia USP. 110p.

Figueiredo, J.L. de \& Menezes, N.A. 2000. Manual de peixes marinhos do sudeste do Brasil - Volume VI (Teleostei 5). São Paulo: Museu de Zoologia USP. $117 p$.

Gobert, B. 1998. Density-dependent size selectivity in Antillean fish traps. Fish. Res. 38: 159-167. 
Hubert, W.A. 1996. Passive capture techniques. In: Murphy B.R. \& Willis, D.W. (eds.) Fisheries techniques. 2nd ed. Bethesda, Maryland: American Fisheries Society. 157-181pp.

Lana, P.C.; Almeida, M.V.O.; Freitas, C.A.F.; Couto, E.C.G.; Conti, L.M.P.; Gonzalez-Peronti, A.L.; Giles, A.G.; Lopes, M.J.S.; Silva, M.H.C. \& Pedroso, L.A. 1989. Estrutura espacial de associações macrobênticas da Gamboa Perequê (Pontal do Sul, Paraná). Nerítica. 4(1,2): 119-136.

Lana, P.C. 2003. As marismas da Baía de Paranaguá: características gerais, modos de apropriação e implicações para a legislação ambiental. Desenvolvimento e Meio Ambiente. 8:11-23.

Lima, G.V. \& Oshiro, L.M.Y. 2002. Aspectos reprodutivos de Palaemon pandaliformis (Stimpson) (Crustacea, Decapoda, Palaemonidae) no Rio Sahy, Mangaratiba, Rio de Janeiro, Brasil. Rev. Bras. Zool. 19(3): 855-860.

Melo, G. A. S. 1996. Manual de identificação dos Brachyura (caranguejos e siris) do litoral brasileiro. $1^{a}$ ed. São Paulo: Plêiade. 603 p.

Melo, G. A. S. 2003. Manual de Identificação dos Crustacea Decapoda de Água Doce do Brasil. São Paulo, Loyola - Centro Universitário São Camilo - Museu de Zoologia da Universidade de São Paulo. 429 p.

Menezes, N.A. \& Figueiredo, J.L. de 1980. Manual de peixes marinhos do sudeste do Brasil - Volume V (Teleostei 4). São Paulo: Museu de Zoologia USP. 106p.

Müller, Y.M.R.; Nazari, E.M.; Bressan, C.M. \& Ammar, D. 1996. Aspectos da reprodução de Palaemon pandaliformis (Stimpson) (Decapoda, Palaemonidae) no manguezal de Ratones, Florianópolis, Santa Catarina. Rev. Bras. Zool. 13(3): 633-642.

Nielsen, L.A. \& Johnson, D.L. 1983. Fisheries Techniques. Blacksburg, Virginia: Southern Printing Company, 468p.

Oliveira, A.; Pinto, T.K.; Santos, D.P.D. \& D'Incao, F. 2006. Dieta natural do siri-azul Callinectes sapidus (Decapoda, Portunidae) na região estuarina da Lagoa dos Patos, Rio Grande, Rio Grande do Sul, Brasil. Iheringia, Sér. Zool. 96(3): 305-313.

Palacios-Sánchez, S.E. \& Vega-Candejas, M.E. 2010. Cambios alimenticios en tres especies de Sphoeroides (Tetraodontiformes: Tetraodontidae) posterior al haracán Isidoro en Bocana de la Carbonera, Sureste del Golfo de México. Rev. Biol. Trop. 58(4): 1223-1235.

Pesserl, B.H. 2007 Seletividade de captura de redes de emalhe em um ambiente de gamboa. Tese de Mestrado. Universidade Federal do Paraná UFPR. 57p.
Ribeiro, O.M. \& Zuanon, J. 2006. Comparação da eficiência de dois métodos de coleta de peixes em igarapés de terra firme da Amazônia Central. ACTA Amazônica. 36(3): 389-394.

Rocha, S.S. 2010. Diferença entre dois métodos de coleta utilizados na captura de crustáceos decápodes em um rio da Estação Ecológica Juréia-Itatins, São Paulo. Iheringia, Sér. Zool. 100(2): 116-122.

Rozas, L.P. \& Minello, T.J. 1997. Estimating densities of small fishes and decapods crustaceans in shallow estuarine habitats: A review of sampling design with focus on gear selection. Estuaries. 20(2): 199-213.

Sanches, E.G. \& Sebastiani, E.F. 2009. Atratores e tempo de submersão na pesca artesanal com armadilhas. Biotemas. 22(4): 199-206.

Sazima, I. 1986. Similarities in feeding behaviour between some marine and freshwater fishes in two tropical communities. J. Fish Biol. 29: 53-65.

Soares, C.R.; Marone, E.; Lessa, G.C.; Lana, P.C.; Lemos, P.B.; Krul, R.; Athayde, S.F. \& Moraes, V.S. 1996. Síntese dos conhecimentos sobre o Rio Perequê, Balneário Pontal do Sul (Paraná), visando a transformação da área num Parque Municipal. Laudo emitido por solicitação do I.A.P. - Instituto Ambiental do Paraná através da SEMA - Secretaria de Estado do Meio Ambiente. 5-8pp.

Stoner, A.W. e Buchanan, B.A. 1990. Ontogeny and overlap in the diets of four tropical Callinectes species. B. Mar. Sci. 46(1): 1-12.

Teixeira, R.R. \& Couto, E. C. G. 2012. Crustacea Decapoda capturados através de coleta passiva em um trecho do Rio dos Mangues (Porto Seguro - BA). Biotemas, 25(4): 149-156.

Uieda, V.S. \& Castro, R.M.C. 1999. Coleta e fixação de peixes de riachos. In: Caramashi, E.P.; Mazzoni, R. \& Peres-Neto, P.R. (eds.) Ecologia de Peixes de Riachos. Rio de Janeiro, Brasil: Série Oecologia Brasiliensis, PPGE-UFRJ. VI. 1-22pp.

Uieda, V.S. \& Uieda, W. 2001. Species composition and spatial distribuition of a stream fish assemblage in the east coast of Brazil: comparison of two field study methodologies. Braz. J. Biol. 61(3): 377-388.

Zar, J. H. 1996. Biostatistical analysis. 3. ed. New Jersey, Prentice Hall. 662p.

Zuur, A.F.; leno, E.N. \& Smith, G.M. 2007. Analysing ecological data. Springer Verlag, Heidelberg. 672p.
Submetido: Fevereiro/2013 Revisado: Fevereiro/2014 Aceito: Março/2014 